\title{
CONSTITUINTES QUÍMICOS DE Licania tomentosa BENTH. (CHRYSOBALANACEAE)
}

\author{
Rachel Oliveira Castilho* \\ Departamento de Produtos Farmacêuticos, Faculdade de Farmácia, Universidade Federal de Minas Gerais, Av. Presidente \\ Antônio Carlos, 6627, Campus UFMG, 31270-901 Belo Horizonte - MG, Brasil \\ Maria Auxiliadora Coelho Kaplan \\ Núcleo de Pesquisas de Produtos Naturais, Centro de Ciências da Saúde, Bloco H, Universidade Federal do Rio de Janeiro, \\ Cidade Universitária, 21941-590 Rio de Janeiro - RJ, Brasil
}

Recebido em 4/12/06; aceito em 21/6/07; publicado na web em 19/12/07

\begin{abstract}
CHEMICAL CONSTITUENTS OF Licania tomentosa BENTH. (CRHYSOBALANACEAE). This paper describes the chemical constituents isolated from leaves and fruits of Licania tomentosa Benth. The plant materials were successively extracted with hexane and methanol. From the extracts the following compounds were obtained: betulinic acid; licanolide, a new triterpene lactone; oleanolic acid, lupeol; palmitoleic and hexadecanoic acid; a mixture of stigmasterol and sitosterol; and a mixture of tormentic, ursolic and betulinic acid. The structures of the natural products were identified on the basis of spectral data.
\end{abstract}

Keywords: triterpene lactone; Licania tomentosa; Chrysobalanaceae.

\section{INTRODUÇÃO}

Licania tomentosa Benth. pertence à família Chrysobalanaceae, ordem Rosales e superordem Rosiflorae (sensu Dalgren, 1980) ${ }^{1}$. Essa família é composta de 17 gêneros e cerca de 450 espécies de hábitos arbustivo e arbóreo distribuídas nas regiões tropicais e subtropicais². No nordeste do Brasil espécies de Licania são conhecidas como oiti ${ }^{3}$ e o óleo extraído de suas sementes é usado como substituto do óleo de "tung" (secante de tintas). Licania tomentosa é muito utilizada na ornamentação de grandes cidades brasileiras ${ }^{4}$.

Na medicina popular, espécies da família Chrysobalanaceae são empregadas para diversos fins: espécies do gênero Parinari são empregadas na África para tratamento da malária'; no Brasil as espécies Chrysobalanus icaco ${ }^{6}$ e Licania rigida ${ }^{7}$ são usadas para tratamento do diabetes, com seus efeitos hipoglicemiante e diurético comprovados farmacologicamente ${ }^{8}$. Os diterpenos encontrados nesta família se mostram de ampla diversidade farmacológica: aqueles obtidos de Parinari curatellifolia mostram-se citotóxicos ${ }^{9}$, enquanto que os encontrados em Chrysobalanus icaco e Parinari capensis têm potencialidade para o tratamento da $\operatorname{SIDA}^{10}$ e ação antifúngica $^{11}$, respectivamente. $\mathrm{O}$ extrato de Licania tomentosa inibe a atividade do vírus herpes-simples ${ }^{12}$ e os triterpenos isolados mostram-se citotóxicos ${ }^{13,14}$. Já os flavonóides de Licania licaniaeflora têm atividade antioxidante ${ }^{15}$, enquanto que o extrato de Licania michauxii induz a morte célular ${ }^{16}$.

O metabolismo especial dessa espécie é representado principalmente por flavonóides, cromonas e triterpenóides ${ }^{17-28}$.

O presente trabalho descreve o estudo químico de L. tomentosa, uma espécie vegetal da qual ainda não se conhece a composição química. $\mathrm{O}$ fracionamento dos extratos e das frações do extrato metanólico de L. tomentosa resultou no isolamento e identificação de ácido betulínico (1); de um triterpeno inédito, licanolídeo ${ }^{29}$ (2); de ácido oleanólico (3); de uma mistura de estigmasterol (4) e sitosterol (5); de lupeol (6); de uma mistura de ácido tormêntico (7), ácido betulínico e ácido ursólico (8) e dois ácido graxos: ácido palmitoléico e hexadecanóico (Figura 1). As estruturas das subs-

*e-mail: roc2006@farmacia.ufmg.br tâncias isoladas foram definidas com base na análise de dados espectroscópicos de IV, EM, RMN ${ }^{1} \mathrm{H}$ e ${ }^{13} \mathrm{C}$ e de experimentos de DEPT, HMBC, HMQC e NOESY. A comparação dos dados obtidos com os registrados na literatura permitiu a confirmação das estruturas dos constituintes químicos isolados ${ }^{30-35}$.

\section{RESULTADOS E DISCUSSÃO}

O estudo químico do extrato hexânico dos frutos de L. tomentosa resultou no isolamento de ácido betulínico (1), licanolídeo (2) e ácido palmitoléico. Os ácidos betulínico (1) e palmitoléico foram identificados por comparação com dados da literatura ${ }^{29,32,35}$. A substância (2) foi denominada de licanolídeo (3ß-hidroxilupano-20,28olídeo), um novo triterpeno lactônico ${ }^{29}$. A fórmula molecular foi determinada, após a metilação, como $\mathrm{C}_{31} \mathrm{H}_{50} \mathrm{O}_{3}$, com base em seu CG/EM. A fragmentação do licanolídeo mostrou sinais com $\mathrm{m} / z$, em 411, 262, 233, 207, 189 e 175, característicos de triterpenóides do tipo lupano ${ }^{34}$. O espectro de absorção na região do infravermelho para o licanolídeo mostrou máximo de absorção em $1763 \mathrm{~cm}^{-1}$, sugerindo a presença de um grupo $\delta$-lactona, além de absorção para o grupo hidroxila em $3524 \mathrm{~cm}^{-1}$. O espectro $\mathrm{RMN}{ }^{1} \mathrm{H}$ para o licanolídeo mostrou sete singletos muito intensos com integração para três hidrogênios em: $\delta 0,87\left(\mathrm{CH}_{3}-26\right), 0,94\left(\mathrm{CH}_{3}-24\right), 0,97$ $\left(\mathrm{CH}_{3}-23\right), 1,02\left(\mathrm{CH}_{3}-25\right), 1,05\left(\mathrm{CH}_{3}-27\right), 1,09\left(\mathrm{CH}_{3}-29\right)$ e $1,15\left(\mathrm{CH}_{3}-\right.$ 30) $\mathrm{ppm}$ referentes a grupos metila ligados a carbono $\mathrm{sp}^{3}$. Esse fato indicou a ausência da ligação dupla entre 20 e 29, característica do ácido betulínico. Em $\delta 3,45$ ppm observou-se um sinal largo referente ao hidrogênio carbinólico (H-3). Em $\delta$ 3,0 e 3,1 ppm observaram-se dois sinais característicos do licanolídeo, referentes aos hidrogênios metínicos ligados ao $\mathrm{C} 9$ e ao $\mathrm{C} 19$, respectivamente. Destacou-se a ausência dos sinais dos hidrogênios vinilidênicos de C29 na região de $\delta$ 4,0-5,0 ppm, característicos do ácido betulínico. No espectro de $\mathrm{RMN}{ }^{13} \mathrm{C}$ do licanolídeo observaram-se dois sinais referentes a carbono carbinólico, um em $\delta 75,8$ ppm e outro em $\delta$ 87,5 ppm. O sinal em $\delta 75,8$ ppm é referente ao átomo de carbono da posição 3 , cuja hidroxila está orientada em estereoquímica $\beta^{30} \mathrm{e}$ o sinal em $\delta 87,5$ ppm é referente ao átomo de carbono da posição 20. No espectro de $\mathrm{RMN}{ }^{13} \mathrm{C}$ do ácido betulínico somente obser- 


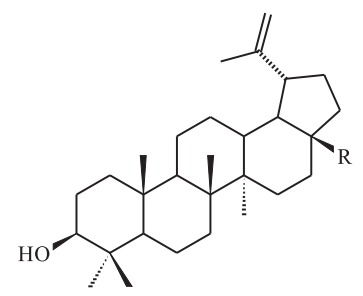

$1 \mathrm{R}=\mathrm{COOH}$

$6 \mathrm{R}=\mathrm{CH}_{3}$<smiles>CC1(C)CCC2(C(=O)O)CC[C@H]3C(=CCC4C5(C)CCC(O)C(C)(C)C5CCC43C)C2C1</smiles>

3

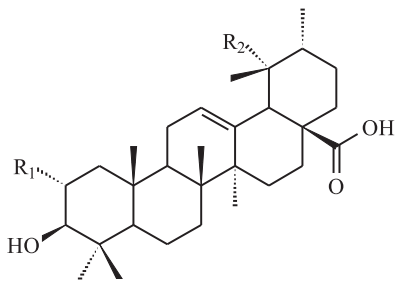

$7 \mathrm{R}^{1}$ e $\mathrm{R}^{2}=\mathrm{OH}$

$8 \mathrm{R}^{1}$ e $\mathrm{R}^{2}=\mathrm{H}$

Figura 1. Constituíntes químicos de Licania tomentosa

vou-se o sinal de carbono carbinólico da posição 3. O espectro de RMN ${ }^{13} \mathrm{C}$ do licanolídeo mostrou também um sinal referente à carbonila ácida em $\delta 179,3$ ppm e ausência dos sinais relativos aos átomos de carbono da ligação dupla entre C20 e C29, característica do ácido betulínico. A determinação mais precisa dos deslocamentos químicos de carbono e hidrogênio para o licanolídeo só foi possível através dos espectros de HMBC e HMQC ${ }^{29}$. O espectro de HMBC permitiu confirmar a formação de uma $\delta$-lactona entre $\mathrm{C} 28$ e C20, pois se observaram correlações entre o átomo de carbono carbinólico em $\delta 87,5$ ppm e os dois grupos metila em C29 e C30, mais desblindadas com $\delta 1,09$ e 1,15 ppm, respectivamente. O grupo isopropila ligado ao C19 no licanolídeo tem configuração $\beta$ para permitir a formação de uma lactona em C20-C28. Experiências de $\mathrm{H}, \mathrm{H}$ NOESY, confirmaram este fato e mostraram efeito overhouse nuclear entre $\mathrm{CH}_{3}-29$ e $\mathrm{CH}-13, \mathrm{CH}_{2}-12 ; \mathrm{CH}_{3}-30$ e $\mathrm{CH}_{2}-21 ; \mathrm{CH}_{3}-27$ e $\mathrm{CH}_{2}-16 ; \mathrm{CH}_{3}-23$ e $\mathrm{CH}_{3}-24$; e entre $\mathrm{CH}_{3}-25$ e $\mathrm{CH}_{3}-26^{29}$.

A fração em AcOEt, resultante da partição do extrato metanólico dos frutos de $L$. tomentosa, foi cromatografada em coluna de gel de sílica, obtendo-se o isolamento do ácido oleanólico, um sólido amarelo pálido (3). Procedeu-se à análise por $\mathrm{RMN}$ de ${ }^{1} \mathrm{H},{ }^{13} \mathrm{C}$, APT, CG/EM (após a metilação) e comparação com dados da lite- ratura para a identificação ${ }^{30}$

O extrato hexânico de folhas de L. tomentosa foi cromatografado em coluna de gel de sílica fornecendo o isolamento de lupeol (6) e de uma mistura de estigmasterol (4) e sitosterol (5). O espectro de massas da fração em Hex/AcOEt a $10 \%$ mostrou $\mathrm{m} / z$ de 412 e 414 que são atribuídos ao estigmasterol e o sitosterol, respectivamente, além de deslocamentos químicos característicos em RMN de ${ }^{1} \mathrm{H}$ e ${ }^{13} \mathrm{C}$ que corroboram com a atribuição para estes dois esteróides ${ }^{31}$. O conjunto de dados espectroscópicos obtidos da fração eluída em Hex/AcOEt a 20\%, um sólido amarelo pálido, permitiu identificálo como lupeol (6). Análise do espectro de RMN ${ }^{1} \mathrm{H}$ desse sólido mostrou seis singletos intensos em $\delta 0,77 ; 0,88 ; 0,97 ; 0,99 ; 1,03$ e 1,31 ppm provenientes dos hidrogênios de grupos metila $(18 \mathrm{H})$. É importante destacar a presença de uma metila adicional nesse espectro quando o mesmo é comparado com espectro do ácido betulínico, que possui uma oxidação na metila número 28 . Foi possível notar também a presença de um singleto em $\delta 1,7 \mathrm{ppm}(3 \mathrm{H})$ referente a grupo metila ligado a carbono $\mathrm{sp}^{2}$, um duplo dubleto em $\delta 3,1 \mathrm{ppm}(1 \mathrm{H}, \mathrm{J}=9,9 \mathrm{~Hz})$ relativo ao hidrogênio carbinólico, além de singletos largos na região de $\delta 4,6$ e 4,7 ppm (2H) pertencentes a dois hidrogênios vinilidênicos. $\mathrm{O}$ espectro de $\mathrm{RMN}{ }^{13} \mathrm{C}$ mostrou a presença de sinais característicos para o lupeol: $\delta 79,73$ (C3), 110,11 (C29) e 151,99 (C20) ppm ${ }^{30}$. Na análise da fração eluída em Hex/AcOEt 30\% identificou-se a mistura de ácido betulínico (1) e ácido hexadecanóico. Após metilação e CG/EM, o cromatograma permitiu concluir que se tratava de uma mistura, contendo uma substância majoritária, o ácido betulínico (1) com tempo de retenção 27,39 min $(97,32 \%)$ e o ácido hexadecanóico, minoritário com tempo de retenção de 13,66 min (2,68\%). Posteriormente, procedeu-se à comparação dos deslocamentos de RMN de ${ }^{1} \mathrm{H},{ }^{13} \mathrm{C}$ e dos dados do cromatograma (CG/EM) com a literatura para a caracterização da mistura ${ }^{30,35}$.

A fração em $\mathrm{CH}_{2} \mathrm{Cl}_{2}$ resultante da partição do extrato metanólico de folhas de L. tomentosa foi cromatografada em coluna de gel de súlica, obtendo-se a mistura de ácido tormêntico, ácido betulínico e ácido ursólico. Após metilação dessa mistura, o cromatograma (CG/ EM) dessa amostra mostrou a presença de dois sinais, com tempo de retenção 74,37 e 76,27 min. Os espectros de massas das substâncias referentes a esses tempos de retenção não apresentaram o sinal do íon molecular, mas apresentaram um sinal com $\mathrm{m} / \mathrm{z}, 262$, característico de esqueletos dos tipos 12-urseno e 20-29 lupeno contendo um substituinte carboximetila na posição 28 , confirmado pela perda de fragmento neutro de 59 u.m.a. A interpretação do espectro de RMN ${ }^{1} \mathrm{H}$ permitiu assinalar muitos sinais referentes a hidrogênios metílicos na região de $\delta$ 0,7-1,5 ppm. Além de sinais referentes a hidrogênios olefínicos, em $\delta 5,1 \mathrm{ppm}$, atribuídos aos hidrogênios ligados ao C12 do ácido ursólico e tormêntico e dois sinais largos atribuídos aos hidrogênios vinilidênicos do ácido betulínico em $\delta$ 4,5 e 4,6 ppm. Observaram-se sinais referentes a hidrogênios carbinólicos em $\delta 3,0$ ppm (m) e sinal referente a hidrogênios ácidos em $\delta 12$ ppm (singleto largo). $\mathrm{O}$ espectro de $\mathrm{RMN}{ }^{13} \mathrm{C}$ mostrou a presença de três substâncias, devido à existência de três sinais de carbonila de grupo carboxila e seis sinais na região de olefinas. A análise desse espectro mostrou sinais característicos para ácido tormêntico (7), ácido betulínico (1) e ácido ursólico $(\mathbf{8})^{30}$.

Estudos químicos com espécies do gênero Licania mostraram, principalmente, o isolamento de flavonóides e triterpenóides. Os flavonóides foram isolados de Licania apetalada, L. licaniaeflora, L. densiflora, L. heteromorpha, L. pittieri, L. pyrifolia e L. carii e são, em sua maioria derivados do campferol, quercetina e miricetina ${ }^{17,18,20,22,23,25-27}$. Já os triterpenóides são do tipo oleanano, ursano e lupano. De L. licaniaeflora isolaram-se ácido oleanólico, ácido maslínico, ácido 3-O-arabinosídeo-oleanólico, ácido betulínico, 
arjunetina, ácido tormêntico, ácido pomólico, entre outros ${ }^{19}$. Já em L. heteromorpha isolaram-se ácido betulínico, derivados do ácido alfitólico e do ácido maslínico ${ }^{21}$. Em $L$. carii isolaram-se os ácidos betulínico, ursólico e maslínico ${ }^{24} \mathrm{e}$ em $L$. pittieri, ácido oleanólico e ursólico $^{27}$. O estudo químico de L. tomentosa, uma espécie vegetal que não apresenta pesquisas quanto a sua composição química, levou ao isolamento e à caracterização de hidrocarbonetos, esteróides e triterpenos, revelando assim uma quantidade grande de derivados da via do acetato-mevalonato, na forma de triterpenos ácidos com esqueletos do tipo oleanano, ursano e lupano, muito característicos do gênero Licania e da família Chrysobalanaceae. Além de um triterpeno inédito do tipo lupano (licanolídeo).

\section{PARTE EXPERIMENTAL}

\section{Procedimentos gerais}

O ponto de fusão foi medido em aparelho do tipo ThomasHoover. A rotação óptica foi realizada no polarímetro Jasco DIP360. As análises por cromatografia com fase gasosa acoplada à espectroscopia de massas (CG/EM) foram realizadas utilizando um cromatógrafo HP 5890 SII acoplado a um espectrômetro de massas Autospect (IE, $70 \mathrm{eV}$ ), equipado com coluna capilar de sílica fundida DB-1 (30 $\mathrm{m} \times 0,20 \mathrm{~mm})$. Hélio foi usado como gás de arraste. Os triterpenos ácidos foram analisados por CG/EM, após metilação com diazometano. Os espectros de RMN ${ }^{1} \mathrm{H}$ e $\mathrm{RMN}{ }^{13} \mathrm{C}$ foram realizados em espectrômetros Varian-Gemini operando em 200 e $50 \mathrm{MHz}$, respectivamente, utilizando solventes deuterados e TMS como padrão interno. As análises cromatográficas em camada fina foram realizadas em gel de sílica $60 \mathrm{~F}_{254}$ e com cromatoplacas de gel de sílica $60 \mathrm{~F}_{254}$ sobre placa de alumínio Merck, de 0,2 mm de espessura. A visualização das substâncias em camada fina foi feita, quando possível, sob luz UV a 254 e $365 \mathrm{~nm}$ e, principalmente, com sulfato cérico.

\section{Material vegetal}

Licania tomentosa foi coletada na cidade do Rio de Janeiro, RJ, em 1997 e identificada pela Dra. R. Fuks do Jardim Botânico do Rio de Janeiro, tendo sido registrada no Herbarium do Museu Nacional, UFRJ, pelo número R195.941.

\section{Extração e isolamento dos constituintes químicos}

As folhas foram secas em estufa com circulação de ar a $40{ }^{\circ} \mathrm{C}$. Após a secagem, o material vegetal foi triturado em moinho de facas. Os frutos foram separados de suas sementes, fragmentados e extraídos frescos.

Esse material botânico foi extraído separadamente por maceração estática a frio com $n$-hexano e metanol até o esgotamento. Os extratos obtidos foram secos sob pressão reduzida em evaporador rotatório e quantificados posteriormente após a remoção total do solvente. O extrato metanólico (40 g), em suspensão com $\mathrm{H}_{2} \mathrm{O}$, foi submetido a partições sucessivas com $n$-hexano, diclorometano, acetato de etila e $n$-butanol.

O extrato hexânico dos frutos $(8 \mathrm{~g})$ foi cromatografado em coluna de gel de sílica, utilizando-se misturas de $n$-hexano, acetato de etila e metanol, além dos solventes puros, como eluente. A fração eluída em Hex/AcOEt 20\% (100 mg) foi purificada por recristalização com pentano, éter de petróleo e diclorometano, obtendo-se três substâncias puras: ácido betulínico (57 mg) (1), licanolídeo (13 mg) (2) e ácido palmitoléico (30 mg). A fração em AcOEt resultante da partição do extrato metanólico dos frutos de
L. tomentosa (1,0 g) foi cromatografada em coluna de gel de sílica, utilizando-se como eluente misturas de $n$-hexano, acetato de etila e metanol em polaridades crescentes. A fração eluída em Hex/AcOEt $30 \%$ (70 mg) foi analisado por CG/EM e por RMN de ${ }^{1} \mathrm{H} \mathrm{e}{ }^{13} \mathrm{C}$, sendo identificada como o ácido oleanólico (3).

O extrato hexânico de folhas de L. tomentosa $(10,0 \mathrm{~g})$ foi cromatografado em coluna de gel de sílica, utilizando-se como eluente misturas de $n$-hexano, acetato de etila e metanol em gradiente de polaridades crescentes, além dos solventes puros. A fração eluída com Hex/AcOEt 10\% rendeu cristais incolores em forma de agulha $(200 \mathrm{mg})$. Esse material foi identificado como sendo uma mistura de estigmasterol (4) e sitosterol (5) (50 mg). A fração eluída em Hex/AcOEt $20 \%$ foi recromatografada em coluna de gel de sílica e a fração eluída em Hex/AcOEt $20 \%$ foi identificada como lupeol (6) (17 mg), já a fração eluída em Hex/AcOEt 30\% rendeu uma mistura de ácido betulínico (1) e ácido hexadecanóico (40 mg). A fração em $\mathrm{CH}_{2} \mathrm{Cl}_{2}$ resultante da partição do extrato metanólico de folhas de L. tomentosa $(6,8 \mathrm{~g})$ foi cromatografada em coluna de gel de sílica, utilizando-se como eluente misturas de $n$-hexano, acetato de etila e metanol em gradientes de polaridades crescentes. A fração eluída em Hex/AcOEt 50\% apresentou-se como um sólido amorfo branco, que foi identificado como uma mistura de ácido tormêntico $(7,9 \%)(7)$, ácido betulínico (79\%) (1) e ácido ursólico $(13,1 \%)(8)(1,1 \mathrm{~g})$

\section{AGRADECIMENTOS}

À Dra. R. Fuks pela identificação botânica e à CAPES e ao CNPq pelo apoio financeiro.

\section{REFERÊNCIAS}

1. Dahlgren, R. M. T.; Bot. J. Linn. Soc. 1980, 80, 91.

2. Brummitt, R. K.; Vascular Plant Families and Genera, Royal Botanic Gardens: Kew, 1992.

3. Braga, R.; Plantas do Nordeste Especialmente do Ceará, $3^{\mathrm{a}}$ ed., Ed. da UFRN: Natal, 1960

4. Rizzini, C. T.; Mors, W. B.; Botânica Econômica Brasileira, Ed. da USP. São Paulo, 1976.

5. Gessler, M. C.; Msuya, D. E.; Nkunya, M. H. H.; Nwasumbi, L. B.; Schär, A.; Heimich, M.; Tanner, M.; J. Ethnopharmacol. 1995, 48, 119.

6. Presta, G. A.; Dissertação de Mestrado, Universidade Federal do Rio de Janeiro, Brasil, 1986.

7. Lacet, M. A. B.; Almeida, J. A.; Almeida, R. N.; Thomas, G.; Resumo do II Simpósio Nacional de Farmacologia e Química de Produtos Naturais, Fortaleza, Brasil, 1983.

8. Presta, G. A.; Pereira, N. A.; Rev. Bras. Farm. 1987, 68, 91.

9. Lee, I-S.; Shamon, L. A.; Chai, H-B.; Chagwedera, T. E.; Bestliman, Y. M.; Farnsworth, N. R.; Cordell, G. A.; Pezzuto, J. M.; Douglas, K. A.; Chem. Biol. Interact. 1996, 99, 193.

10. Gustafson, K. R.; Munro, M. H. G.; Blunt, J. W.; Cardellina, J. H.; Mc Malon, J. B.; Gulakowshi, R. J.; Cragg, G. M.; Cox, P. B.; Linda, S. J.; Tetrahedron 1991, 47, 4547.

11. Garo, E.; Maillard, M.; Hostettmann, K.; Stoeckli-Evvans, H.; Mavi, S.; Helv. Chim. Acta 1997, 80, 538.

12. Miranda, M. M. F. S.; Gonçalves, J. L. S.; Romanos, M. T. V.; Silva, E. P.; Pinto, L.; Silva, M. H.; Ejzemberger, R.; Granja, L. F. Z.; Wigg, M. D.; Phytomedicine 2002, 9, 641 .

13. Fernandes, J.; Castilho, R. O.; Costa, M. R. da; Wagner-Souza, K.; Kaplan, M. A. C.; Gattass, C. R.; Cancer Lett. 2003, 190, 165.

14. Fernandes, J.; Weinlich, R.; Castilho, R. O.; Kaplan, M. A. C.; AmaranteMendes, G. P.; Gattass, C. R.; Cancer Lett. 2005, 219, 49.

15. Braça, A.; Sortino, C.; Politi, M.; Morelli, I.; Mendez, J.; J. Ethnopharmacol. 2002, 79, 379.

16. Badisa, R. B.; Chaudhuri, S. K.; Pilarinou, E.; Ritkoski, N. J.; Hare, J.; Levenson, C. W.; Cancer Lett. 2000, 149, 61.

17. Castilho, R. O.; Tese de Doutorado, Universidade Federal do Rio de Janeiro, Brasil, 2001.

18 Braca, A.; Luna, D.; Mendez, J.; Morelli, I.; Biochem. Syst. Ecol. 2002, $30,271$. 
19. Braca, A.; Sortino, C.; Mendez, J.; Morelli, I.; Fitoterapia 2001, 72, 585.

20. Braca, A.; Bilia, A. R.; Mendez, J.; Morelli, I.; Fitoterapia 2001, 72, 182.

21. Braca, A.; Bilia, A. R.; Mendez, J.; Morelli, I.; Biochem. Syst.and Ecol. 1999, 27, 527.

22. Braca, A.; Bilia, A. R.; Mendez, J.; Morelli, I.; Phytochemistry 1999, 51, 1125.

23. Braca, A.; De Tommasi, N.; Mendez, J.; Morelli, I.; Pizza, C.; Phytochemistry 1999, 51, 1121.

24. Bilia, A. R.; Mendez, J.; Morelli, I.; Pharm. Acta Helv. 1996, 71, 191.

25. Bilia, A. R.; Morelli, I.; Mendez, J.; J. Nat. Prod. 1996, 59, 297.

26. Bilia, A. R.; Ciampi, L.; Mendez J.; Morelli, I.; Pharm. Acta Helv. 1996, $71,199$.

27. Mendez, J.; Bilia, A. R.; Morelli, I.; Pharm. Acta Helv. 1995, 70, 223.
28. Carvalho, M. G.; Cândido, L. F. O.; Costa, P. M.; Rumjanek, V. M.; Nat. Prod. Res. 2005, 19, 7.

29. Castilho, R. O.; Oliveira, R. R.; Kaplan, M. A. C.; Fitoterapia 2005, 76, 562.

30. Mahato, S. B.; Kundu, A. P.; Phytochemistry 1994, 37, 1517.

31. Koizumi, N.; Fujimoto, Y.; Takeshita, T.; Ikekawa, N.; Chem. Pharm. Bull. 1979, 27, 38 .

32. Mc Lafferty, F. W.; Stanfer, D. B.; Registry of Mass Spectral Data, WilleyInterscience Publication: New York, 1989.

33. Begum, S.; Sultana, R.; Siddiqui, B. S.; Phytochemistry 1997, 44, 329.

34. Villar, A.; Payá, M.; Hortigüela, M. D.; Cortes, D.; Planta Med. 1986, 1, 43.

35. Levy, G. C.; Topics in Carbon-13 NMR Spectroscopy, Wiley-Interscience Publication: New York, 1976. 